\title{
Compressor fault diagnosis based on SVM and GA
}

\author{
Milad Golmoradi ${ }^{1}$, Ebrahim Ebrahimi ${ }^{2}$, Mohamad Javidan ${ }^{3}$ \\ Department of Mechanical Engineering, College of Engineering, Kermanshah Branch, \\ Islamic Azad University, Kermanshah, Iran \\ ${ }^{1}$ Corresponding author \\ E-mail: 1'golmoradi17@gmail.com,2e.ebrahimi57@gmail.com,3javidan833@gmail.com
}

Received 26 March 2017; accepted 12 April 2017

DOI https://doi.org/10.21595/vp.2017.18392

Check for updates

Abstract. Due to growth of mechanization and automation, today's industrial systems are becoming more complex. A small breakdown of any non-redundant machine component affects the operation of the entire system. Compressors are utilized widely in the oil and chemical industry. Great attention has been paid to the condition monitoring and fault diagnosis of the Compressor by the field engineers and technicians. In this study, an effective and reliable method based on vibration analysis and with signal processing and classification techniques is presented for troubleshooting of a centrifugal Compressor. Among different time - frequency methods, wavelet transformation extracts information about the signal time scale through a series of convolution operation between the measured signals and the basis wavelet which was used as a preprocessing. The used mother wavelet is (db4) in which the original signal is switched to multiple details signals; then it features are taken from pre-processed signals and they were introduced to support vector machines as input. Kernel function used here in the support vector machine is RBF in which the parameters of support vector machine were optimized using Genetic Algorithm for better performance to increase the accuracy of classification. The highest accuracy was obtained as $100 \%$. The obtained accuracy clearly indicates high safety margin of the multistage centrifugal pump for fault detection.

Keywords: centrifugal compressor, fault diagnosis, SVM, GA, blade.

\section{Introduction}

Compressors are common systems in industries of the new century. The problem of increase in pressure in different industrial processes, especially petrochemical processes, is highly significant. On the other hand, confidence in proper operation is always a significant problem raised during application of industrial equipment. Therefore, condition monitoring systems have drawn increasing attention to themselves. Considering significant maintenance cost of compressors in relation to their total cost, advanced methods of condition monitoring should be adopted for maximization of these costs [1]. Every dynamic, electric or thermal system has definite indicators which typically show normal condition of a system during operation. Changes of these indicators, even insignificant ones, could signify problems or failure of the system. Therefore, condition monitoring refers to determining condition of the system by measuring its characteristic indicators followed by usage of this information for prediction of future system failure [2]. Condition monitoring is done through different methods. Each method emphasizes certain indicators of operational condition of a system, measures distinctive parameters, and communicates health of the system [3]. In vibration analysis technique, vibrations of compressor are measured by special sensors. As a result, one could analyze vibrations of the system to define its problems and defects. In addition, vibration analysis could be used to troubleshoot the machines [4]. Today, increase of accuracy and reduction of error caused by human judgment should be done through modeling methods so as to enable further troubleshooting and failure detection. In this regard, numerous modeling methods have been suggested among which one could point to fuzzy logic (or ANFIS) [5], artificial neural networks (ANN) [6], and support vector machine (SVM) [7]. Support vector machine is one of the relatively new methods used for classification since it shows higher efficiency than older methods of classification such as artificial neural network. In the case of using support vector machine, linear data classification is done. In the case of linear 
data division, a line is selected that offers higher confidence. Solving the equation for finding optimal line of data is done via second-degree programming methods which are common methods of solving limited problems. Considering the fact that support vector machine acts in a binary manner and it can conduct binary classification, multi-class SVM classification method is used to overcome the limitation in situations where there are more than two classes [8]. In order to improve result of SVM classification when talking about optimization, one should use different meta-heuristic algorithm. By using this algorithm and defining objective function for the algorithm, one could improve the results of classification and accuracy of classification [9]. The objective of present study is to use genetic algorithm for optimization of SVM parameters so as to classify failures of compressor based on vibration spectrum analysis.

\section{Materials and methods}

In present project, a gas compressor installed in gas compressor station of Iranian Gas Transmission Company was tested. The most critical failures of compressors occur in their blades. That is why present study is concerned with fracture and erosions of compressors' blades of Iranian Gas Transmission Company. If detection system is developed sufficiently, it will be able to detect small failures.

Table 1. Specifications of compressor

\begin{tabular}{|c|c|c|}
\hline \multicolumn{2}{|c|}{ Type of compressor } & Ten-step turbo-compressor \\
\hline Power & $(\mathrm{kW})$ & 10340 \\
\hline Airflow rate & $(\mathrm{kg} / \mathrm{s})$ & 48 \\
\hline Normal speed & $(\mathrm{rpm})$ & 11900 \\
\hline Critical speed & $(\mathrm{rpm})$ & 22800 \\
\hline Weight & $(\mathrm{kg})$ & 29000 \\
\hline
\end{tabular}

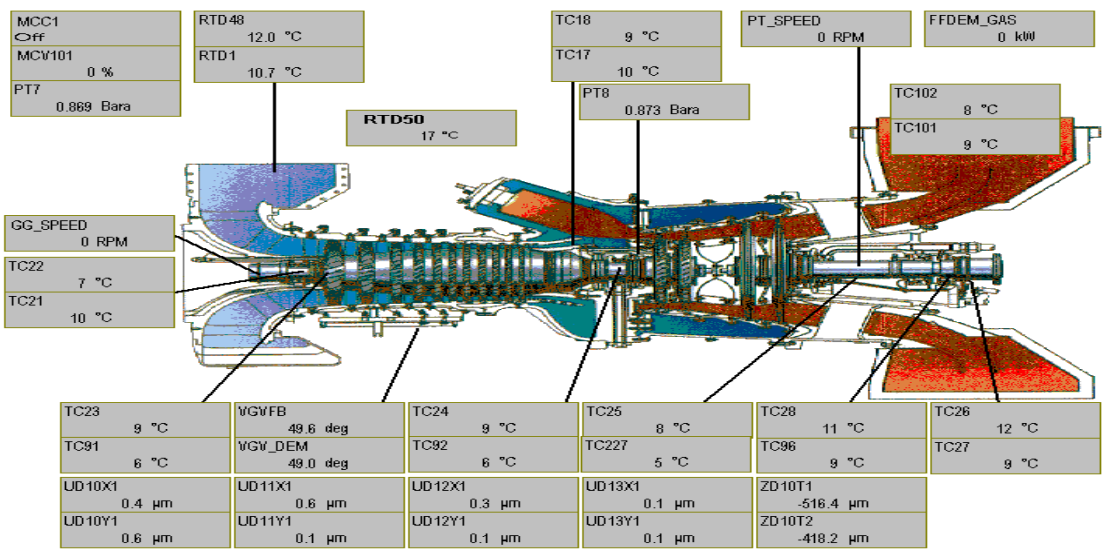

Fig. 1. Schematic representation of compressor installed in Iranian Gas Transmission Company and some of its characteristics

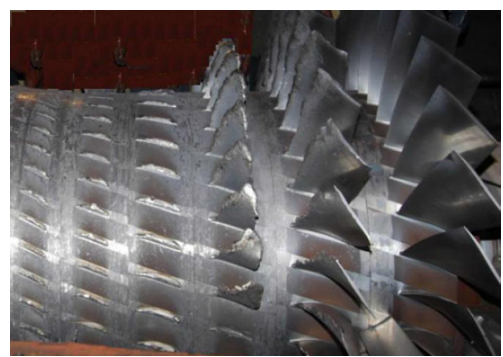

Fig. 2. Broken and worn blades 
In the present survey, registration of vibration data is done through vibration measurement and registration system. In addition, unidirectional piezoelectric accelerometer was used as vibration sensor.

\subsection{Signal processing}

Before analyzing main signals to extract features more properly, a pre-processing or wavelet transform was done on main signals. On all record signals, all healthy and failing states of discrete wavelet analysis was conducted. After analysis of signals and review of literature of previous studies, a discrete wavelet was used as main one. The wavelet is of Daubechies 4 wavelet (db4) type (with three levels) which was applied on all experimental treatments. After applying Daubechies wavelet on signals, coefficients of details and approximation of significant characteristics were used for data analysis so as to distinguish signal behaviors from each other [10]. After applying the mother wavelet on signals, statistical characteristics of vibration spectra were extracted through coefficients of details and approximation. Some functions used in condition monitoring and extraction of features are represented in Table 2.

Table 2. Statistical functions for extraction of features

\begin{tabular}{|c|c|}
\hline Statistic & Statistical functions \\
\hline Mean & $F_{1}=\frac{\sum_{n=1}^{N} x(n)}{N}$ \\
\hline Standard deviation & $F_{2}=\sqrt{\frac{\sum_{n=1}^{N}\left(x(n)-F_{1}\right)^{2}}{N}}$ \\
\hline $\begin{array}{l}\text { Root mean square } \\
\text { of signal value }\end{array}$ & $F_{3}=\left(\frac{\sum_{n=1}^{N} \sqrt{|x(n)|}}{N}\right)^{2}$ \\
\hline Root mean square & $F_{4}=\sqrt{\frac{\sum_{n=1}^{N}(x(n))^{2}}{N}}$ \\
\hline $\begin{array}{l}\text { Peak amplitude } \\
\text { of signal }\end{array}$ & $F_{5}=\max (|x(n)|)$ \\
\hline Steepness factor & $F_{6}=\frac{F_{5}}{F_{4}}$ \\
\hline Variance & $F_{7}=\frac{\sum_{n=1}^{N}\left(x(n)-F_{1}\right)^{2}}{N}$ \\
\hline Harmonic mean & $F_{8}=\frac{N}{\sum_{n=1}^{N} \frac{1}{x(n)}}$ \\
\hline $\begin{array}{c}\text { Coefficient } \\
\text { of dispersion }\end{array}$ & $F_{9}=\frac{F_{2}}{F_{1}} \times 100$ \\
\hline $\begin{array}{l}\text { Average deviation } \\
\text { of mean }\end{array}$ & $F_{10}=\frac{\sum_{n=1}^{N}\left|x(n)-F_{1}\right|}{N}$ \\
\hline Slip & $F_{11}=\frac{\frac{1}{N} \sum_{n=1}^{N}\left(x(n)-F_{1}\right)^{3}}{\left(\sqrt{\frac{1}{N} \sum_{n=1}^{N}\left(x(n)-F_{1}\right)^{2}}\right)^{3}}$ \\
\hline Skewness & $F_{12}=\sqrt{\frac{N-1}{N}} \times \frac{1}{(N-2) \cdot F_{12}^{1.5}} \times \sum_{n=1}^{N}\left(x(n)-F_{1}\right)^{3}$ \\
\hline Kurtosis & $F_{13}=\frac{(N-1) \times(N+1)}{(N-3) \times(N-2) \times N \times{F_{12}}^{2}} \times \sum_{n=1}^{N}\left(x(n)-F_{1}\right)^{4}-\frac{3 \times(N-1)^{2}}{(N-2)(N-3)}+3$ \\
\hline
\end{tabular}




\subsection{Selection of features}

After selection of features mentioned in Table 2, more useful features had to be selected for classification since the features were many in number. To do this, WEKA Software was used and superior characteristics were selected to be transferred to encoding section.

\subsection{Data classification}

In In this part of present study, selection of required characteristics was followed by classification of experimental data through SVM classifier. In this study, the strategy of "one against all" was used which is one of the significant strategies of multi-class support vector machine classification.

Out of vibration signal data, all experimental states were developed after selection of top features. The generated Access file was used as input to SVN network for consequent classification. In the present study, RBF cornel (i.e. radial basis function cornel) was used as core function of support vector machine. The parameters of weight, bias, and Zigma of core-related parameter were given to genetic algorithm for optimization.

The parameters selected for SVM optimization in genetic algorithm include Gaussian mutation function, maximum generation (10.000), combination rate (0.8) and size of population (200).

\section{Discussion and results}

The review of vibration signals of different states Fig. 3 permits one to conclude that there is an intangible change between vibration spectra of healthy state with failure state of a set. Because of insignificant change of vibration spectrum, one cannot detect failure through shape of recorded signals. However, further analysis and processing should be done for troubleshooting.

\subsection{Selection of features}

After applying wavelet transform on vibration signals, WEKA Software was used to select top features because of two reasons. First, volume of data increases due to division of main signal into approximation signals and higher details. Second, prevention from adding low-value features to support vector machine is needed. Because 13 features were used for extraction of data from main signal, application of $\mathrm{db} 4$ wavelet transform at three levels (including three signals of details and one signal of approximation) let to determination of 72 features. In the case of selecting these features, total number of features at time-frequency domain was 8 . This number of features signifies high information value and it contributes to SVM-based classification significantly.

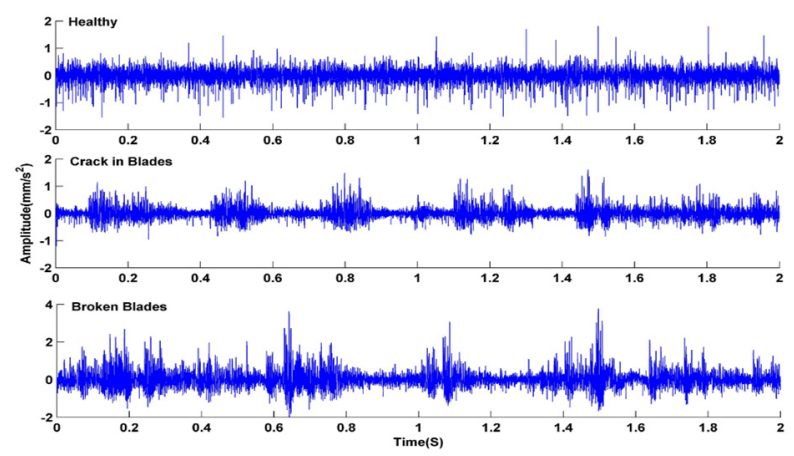

Fig. 3. Vibration signals in different states of compressor

After selection of top features, selected features were added to support vector machine. In the present study, "one against all" method was adopted and as mentioned before some parameters of 
support vector machine were optimized for obtaining better classification and higher accuracy of genetic algorithm. In the following table, classification confusion matrix developed by SVM and genetic algorithm is represented.

Table 3. Confusion matrix of testing final model for different states of signal analysis

\begin{tabular}{|c|c|c|c|}
\hline Broken blades & Crack in blades & Healthy & Classification label \\
\hline 0 & 0 & 15 & Healthy \\
\hline 0 & 15 & 0 & Crack in blades \\
\hline 15 & 0 & 0 & Broken blades \\
\hline
\end{tabular}

\section{Conclusions}

The objective of present study is smart, accurate and quick detection of different types of failures of compressor. Vibration data of compressor was collected in healthy and failure states through piezoelectric accelerometer sensor and data acquisition system. In the present survey, a method was developed for condition monitoring of compressor based on support vector machine (SVM). The results of classification suggested that accuracy of introduced method in troubleshooting of compressor was 100 percent.

\section{References}

[1] Yunsong L., Wang F., Jia M., Qi Y. Centrifugal compressor fault diagnosis based on qualitative simulation and thermal parameters. Mechanical Systems and Signal Processing, Vol. 81, 2016, p. 259-273.

[2] Bengtsson M. Condition based maintenance of technical systems. Conference Proceedings: 17th Congreso Europeo de Mantenimiento, 2004, p. 147-156.

[3] Kostyukov V. N., Kostyukov A. V., Boychenko C. H. Intelligent machinery condition monitoring based on adaptive measurements. Procedia Engineering, Vol. 152, 2016, p. 527-530.

[4] Mahmood S. T. Use of Vibrations Analysis Technique in Condition Based Maintenance. Master of Science Thesis in the Master Degree Programme Production Engineering and Management, 2010.

[5] Pandey V. P., Choudhary P. C. Induction motor condition monitoring using fuzzy logic. Advance in Electronic and Electric Engineering, Vol. 3, Issue 6, 2013, p. 755-764.

[6] Rafieea J., Arvania F., Harifi A., Sadeghic M. H. Intelligent condition monitoring of a gearbox using artificial neural network. Mechanical Systems and Signal Processing, Vol. 21, Issue 4, 2007, p. 1746-1754.

[7] Fafa C., Baoping T., Renxiang C. A novel fault diagnosis model for gearbox based on wavelet support vector machine with immune genetic algorithm. Measurement, Vol. 46, 2013, p. 220-232.

[8] Pal Mahesh Multiclass approaches for support vector machine based land cover classification. MapIndia Conference, 2005, p. 1-16.

[9] Nezamabadi-pour H. Genetic Algorithm Basic Concepts and Advanced Topics. Shahid Bahonar University, Vols. 1-4, 2011.

[10] Mallat S. A Wavelet Tour of Signal Processing. 3 Edition, Academic Press, the Sparse Way, 2009.

[11] Farokhzad S., Ahmadi H., Jaefari A., Asadi Asad Abad M. R., Ranjbar Kohan M. Artificial neural network based classification of faults in centrifugal water pump. Journal of Vibroengineering, Vol. 14, Issue 4, 2012, p. 1734-174. 